\title{
Clinicopathological and Molecular Differences Between Gastric-type Mucinous Carcinoma and Usual-type Endocervical Adenocarcinoma of the Uterine Cervix
}

\author{
HERA JUNG ${ }^{1,2}$, GO EUN BAE ${ }^{3}$, HYE MIN KIM $^{4}$ and HYUN-SOO KIM ${ }^{1}$ \\ ${ }^{1}$ Department of Pathology and Translational Genomics, Samsung Medical Center, \\ Sungkyunkwan University School of Medicine, Seoul, Republic of Korea; \\ ${ }^{2}$ Department of Pathology, CHA Ilsan Women's and Children's General Hospital, \\ CHA University, Goyang, Republic of Korea; \\ ${ }^{3}$ Department of Pathology, Chungnam National University Hospital, Chungnam \\ National University School of Medicine, Daejeon, Republic of Korea; \\ ${ }^{4}$ Department of Pathology, Yonsei University College of Medicine, Seoul, Republic of Korea
}

\begin{abstract}
Background/Aim: We investigated differences in the clinicopathological and molecular characteristics between gastric-type mucinous carcinoma (GMC) and usualtype endocervical adenocarcinoma (UEA). Patients and Methods: We collected the clinicopathological information and performed targeted genomic sequencing analysis. Results: GMCs exhibited significantly deeper invasion depth, larger horizontal spread, more advanced stage, more frequent distant metastasis, and more frequent parametrial and vaginal extension. Disease-free survival time of GMC patients was significantly shorter than that of UEA patients. GMCs displayed mutant p53 immunostaining pattern, whereas UEAs exhibited p16 block positivity. GMCs harbored mutations in KRAS, TP53, NF1, CDKN2A, STK11, and ARIDIA. One GMC exhibited MDM2 amplification. In contrast, UEAs harbored mutations in HRAS, PIK3CA, and BRCA2. Two UEAs were found to have novel TP53 mutations. Conclusion: GMC is associated with more aggressive behavior than UEA. Distinctive p53 and p16
\end{abstract}

This article is freely accessible online.

Correspondence to: Hyun-Soo Kim, Department of Pathology and Translational Genomics, Samsung Medical Center, Sungkyunkwan University School of Medicine, 81, Irwon-ro, Gangnam-gu, Seoul 06351, Republic of Korea. Tel: +82 234101243, Fax: +82 234142831, e-mail: hyun-soo.kim@samsung.com; Hye Min Kim, Department of Pathology, Yonsei University College of Medicine, 51, Yonsei-ro, Seodaemun-gu, 03722 Seoul, Republic of Korea. Tel: +82 3151898252, Fax: +82 3151898247, e-mail: pinkmin15@yuhs.ac

Key Words: Uterus, cervix, usual-type endocervical adenocarcinoma, gastric-type mucinous carcinoma, immunohistochemistry, targeted sequencing. immunostaining patterns enable differential diagnosis. GMC and UEA exhibit genetic heterogeneity with potentially actionable molecular alterations.

Cervical cancer is the fourth most common gynecological malignancy and the fourth leading cause of cancer-related death in women worldwide $(1,2)$, although cervicovaginal cytology screening decreased the incidence rate of cervical cancer and its associated mortality rate. Adenocarcinoma of the uterine cervix is relatively less common than squamous cell carcinoma and accounts for approximately $10-20 \%$ of all cervical cancers $(3,4)$. However, despite the declined incidence of cervical cancer, the proportion of endocervical adenocarcinoma has increased (4-6). The International Endocervical Adenocarcinoma Criteria and Classification (IECC) is a recent system to classify endocervical adenocarcinoma into human papillomavirus (HPV)-associated adenocarcinoma (HPVA) and non-HPV-associated adenocarcinoma (NHPVA) categories based on morphological features (7). Identification of high-risk HPV, notable mitotic activity, and numerous apoptotic bodies across the glandular lumina allowed diagnosing HPVAs. The HPVAs were further divided into usual type, mucinous type (intestinal type, signet ring cell type, and not otherwise specified), villoglandular type, and invasive stratified mucin-producing carcinoma (invasive stratified mucin-producing intraepithelial lesion), depending on their cytoplasmic mucin component and nuclear characteristics. Meanwhile, NHPVAs include gastric-type mucinous carcinoma (GMC), mesonephric carcinoma, serous carcinoma, clear cell carcinoma, and endometrioid adenocarcinoma. Among these carcinomas, GMC, which is the second most common subtype of endocervical adenocarcinoma, is included in the 2014 World Health Organization (WHO) Classification of Tumours of Female Reproductive Organs (1). Although the incidence of 
GMC remains low, it is diagnosed after it progresses into an advanced disease and presents an aggressive clinical course (7). In addition to the clinical outcome, two entities exhibit different pathogenetic mechanisms. HPV infection has been shown to be not involved in the pathogenesis of GMC, in which p16 expression is negative or patchy positive (8).

Due to its rarity, clinicopathological and molecular characteristics of GMC are seldom reported. Despite certain recent studies that present the morphological features and targeted sequencing data of GMC (9), the problem of distinguishing GMC from HPVA remains unresolved. In this study, we analyzed and compared the clinicopathological and molecular characteristics of GMC to those of usual-type endocervical adenocarcinoma (UEA).

\section{Patients and Methods}

Case selection. This retrospective study (4-2018-1138) was reviewed and approved by the Institutional Review Board at the Severance Hospital (Seoul, Republic of Korea). The study population comprised 31 consecutive patients who had undergone hysterectomy and been histologically diagnosed as having invasive endocervical adenocarcinoma at Severance Hospital (Seoul, Republic of Korea) from April 2015 to June 2016. All available hematoxylin and eosin-stained sections were reviewed by two board-certified pathologists specialized in gynecologic oncology (H.M.K. and H.-S.K.). All the histological subtypes were determined according to the WHO Classification (1). Two, two, one, one, and one patients with clear cell carcinoma, mesonephric carcinoma, carcinosarcoma, signet-ring cell carcinoma, and serous carcinoma were excluded. None of the patients was diagnosed as having endometrioid carcinoma of the uterine cervix. Two patients who had received preoperative chemotherapy or concurrent chemoradiation therapy were excluded. Two patients for whom no specimens were suitable for immunostaining were excluded. Finally, we extracted eight cases of GMC and 10 cases of UEA.

Clinical and pathological information, including the age of patients at initial diagnosis, type of surgical treatment, depth of invasion, largest horizontal spread value, endomyometrial extension, parametrial extension, vaginal extension, adnexal extension, involvement of the vaginal and parametrial resection margins, lymphovascular and perineural invasion, lymph node metastasis, initial International Federation of Gynecology and Obstetrics (FIGO) stage, local recurrence, distant metastasis, disease-free survival (DFS), and overall survival (OS), was obtained from the electronic medical record system and pathology reports. The invasion depth and largest horizontal spread were measured directly on the surgical specimen. Follow-up data after surgery are available for all patients. Local recurrence and distant metastasis were revealed based on imaging analyses, including computed tomography and magnetic resonance imaging. In order to analyze DFS, the primary endpoint was defined as the time of local recurrence or distant metastasis - whichever occured first.

Immunohistochemical staining. Immunostaining was performed using an automatic instrument [Ventana Benchmark XT (Ventana Medical Systems, Oro Valley, AZ, USA)] (10-18). Antigen retrieval was performed using the Cell Conditioning Solution $(\mathrm{CC} 1$, Ventana
Medical Systems). The 4- $\mu$ m-thick formalin-fixed paraffinembedded (FFPE) sections were incubated with primary antibodies against p16 (prediluted, clone E6H4; Ventana Medical Systems) and p53 (1:300, clone DO-7; Novocastra, Leica Biosystems, Newcastle Upon Tyne, UK). After chromogenic visualization using an ultraView Universal DAB Detection Kit (Ventana Medical Systems), sections were counterstained with hematoxylin. Appropriate positive and negative controls were concurrently stained to validate the staining method. Endometrial serous carcinoma and ovarian high-grade serous carcinoma were used as positive controls for p16 and p53 expression, respectively. Negative controls were prepared by substituting the non-immune serum for primary antibodies, which resulted in no detectable staining. The p53 immunostaining patterns were interpreted as either missense mutation, nonsense mutation, or wild-type patterns when p53 expression was diffused and strong ( $>60 \%$ of tumor cell nuclei), completely absent $(0 \%)$, or focal and weakly positive, respectively $(11,14,16,18,19)$. The p16 immunostaining pattern was interpreted as block-positive when p16 expression was strong and horizontally continuous, and included nuclear or nuclear-pluscytoplasmic staining. All other p16 immunostaining patterns, including focal or wispy nuclear staining, and blob-like, puddled, or scattered cytoplasmic staining were interpreted as patchy positive $(13,14,19,20)$.

$H P V$ genotyping. We performed a polymerase chain reaction (PCR)based microarray for HPV genotyping, using a commercially available HPV 9G DNA chip (BMT HPV 9G DNA Chip, Biometrix Technology, Chuncheon, Republic of Korea) (20-22). The 9G test examined the presence of 14 high-risk $(16,18,31,33,35,39,45$, $51,52,56,58,59,66,68)$ and 5 low-risk $(6,11,34,40,42) \mathrm{HPV}$ types. The PCR mixture consisted of $10 \mu \mathrm{l}$ of the extracted target DNA, $10 \mu \mathrm{l}$ of the primer set (provided by the manufacturer), and a PCR premix (provided by the manufacturer), which contained dNTP and Taq DNA polymerase in an amplification buffer. Amplification was performed using the following steps: predenaturation for $5 \mathrm{~min}$ at $94^{\circ} \mathrm{C} ; 4030$-s denaturation cycle at $94^{\circ} \mathrm{C}$; 4030 -s annealing cycle at $45^{\circ} \mathrm{C} ; 4030$-s elongation cycle at $72^{\circ} \mathrm{C}$; and a final 5-min elongation step at $72^{\circ} \mathrm{C}$. PCR products were electrophoresed in a $2 \%$ agarose gel to confirm successful amplification. Each hybridization chamber of the $9 \mathrm{G}$ was covered with a mixture of the hybridization solution $(35 \mu \mathrm{l})$ and PCR product $(15 \mu \mathrm{l})$, followed by incubation at $23-26^{\circ} \mathrm{C}$ for $30 \mathrm{~min}$. After washing, array images were scanned and imaged using a fluorescent scanner (ScanArray GX Microarray Scanner, PerkinElmer Life and Analytical Sciences, Waltham, MA, USA).

Targeted sequencing. Histological confirmation for the presence of tumor tissue representing at least $20 \%$ of the nucleated cells on the H\&E-stained glass slides was performed by a single board-certified gynecological pathologist (H.-S.K.), who also marked the densest tumor area avoiding as much as possible necrotic and hemorrhagic areas. Ten-micrometer tissue sections were cut from FFPE blocks. The marked areas were subsequently macrodissected with a scalpel and brought into $1.5 \mathrm{ml}$ tubes for tissue digestion. Genomic DNA were extracted using a QIAamp DSP DNA FFPE Tissue Kit (Qiagen, Valencia, CA, USA). The quantity of the extracted DNA was measured by the Qubit 2.0 Fluorometer (Life Technologies, Grand Island, NY, USA). Mutational and copy number analyses were performed using the TruSight Tumor 170 Kit (Illumina, San Diego, CA, USA) panel (23). 
Jung et al: Gastric-type Mucinous Carcinoma and Usual-type Endocervical Adenocarcinoma

Table I. Gene list for TruSight Tumor 170.

\begin{tabular}{|c|c|c|c|c|c|c|c|}
\hline \multicolumn{8}{|c|}{ Small nucleotide variant and indels (from DNA) } \\
\hline$A K T 1$ & $C C N D 1$ & EP300 & $F G F 7$ & $I D H 1$ & MSH2 & PAX7 & RET \\
\hline$A K T 2$ & $C C N D 2$ & $E R B B 2$ & $F G F 8$ & $I D H 2$ & MSH3 & PDGFRA & RICTOR \\
\hline AKT3 & CCNE1 & ERBB3 & $F G F 9$ & $I N P P 4 B$ & MSH6 & $P D G F R B$ & ROS1 \\
\hline$A L K$ & $C D 79 A$ & $E R B B 4$ & FGF10 & $J A K 2$ & MTOR & $P I K 3 C A$ & RPS6KB1 \\
\hline$A P C$ & $C D 79 B$ & ERCC1 & $F G F 14$ & $J A K 3$ & MUTYH & $P I K 3 C B$ & SLX4 \\
\hline$A R$ & $\mathrm{CDH1}$ & ERCC2 & $F G F 23$ & $K D R$ & $M Y C$ & $P I K 3 C D$ & $S M A D 4$ \\
\hline ARID1A & $C D K 12$ & $E R G$ & $F G F R 1$ & $K I T$ & MYCL1 & PIK $3 C G$ & SMARCB1 \\
\hline$A T M$ & $C D K 4$ & ESR1 & $F G F R 2$ & $K M T 2 A$ & $M Y C N$ & $P I K 3 R 1$ & $S M O$ \\
\hline$A T R$ & CDK6 & $E Z H 2$ & $F G F R 3$ & $K R A S$ & $M Y D 88$ & $P M S 2$ & $S R C$ \\
\hline$B A P 1$ & $C D K N 2 A$ & $F A M 175 A$ & FGFR4 & $M A P 2 K 1$ & $N B N$ & $P P P 2 R 2 A$ & STK11 \\
\hline BARDI & CEBPA & FANCI & FLT1 & $M A P 2 K 2$ & $N F 1$ & PTCH1 & $T E R T$ \\
\hline$B C L 2$ & CHEK1 & FANCL & FLT3 & $M C L 1$ & NOTCH1 & PTEN & TET2 \\
\hline BCL6 & CHEK2 & $F B X W 7$ & FOXL2 & $M D M 2$ & NOTCH2 & PTPN11 & TMPRSS2 \\
\hline$B R A F$ & $C R E B B P$ & $F G F 1$ & GEN1 & MDM4 & NOTCH3 & RAD51 & TP53 \\
\hline BRCAl & $C S F 1 R$ & $F G F 2$ & GNA11 & MET & $N P M 1$ & $R A D 51 B$ & TSC1 \\
\hline$B R C A 2$ & CTNNB1 & $F G F 3$ & $G N A Q$ & MLH1 & $N R A S$ & $R A D 51 C$ & TSC2 \\
\hline$B R I P 1$ & $D D R 2$ & $F G F 4$ & GNAS & MLLT3 & $N R G 1$ & $R A D 51 D$ & $V H L$ \\
\hline BTK & DNMT3A & $F G F 5$ & HNF1A & $M P L$ & $P A L B 2$ & $R A D 54 L$ & $X R C C 2$ \\
\hline CARD11 & $E G F R$ & FGF6 & HRAS & MRE11A & PAX3 & $R B 1$ & \\
\hline \multicolumn{8}{|c|}{ Copy number variation (from DNA) } \\
\hline$A K T 2$ & $C C N D 3$ & ERBB3 & $F G F 5$ & $F G F 23$ & $L A M P 1$ & $N R G 1$ & RICTOR \\
\hline$A L K$ & CCN21 & ERCC1 & FGF6 & FGFR1 & $M D M 2$ & PDGFRA & RPS6KB1 \\
\hline$A R$ & $C D K 4$ & ERCC2 & $F G F 7$ & $F G F R 2$ & MDM4 & $P D G F R B$ & TFRC \\
\hline ATM & CDK6 & ESR1 & $F G F 8$ & FGFR3 & $M E T$ & $P I K 3 C A$ & \\
\hline$B R A F$ & CHEK1 & $F G F 1$ & $F G F 9$ & FGFR4 & $M Y C$ & $P I K 3 C B$ & \\
\hline BRCAl & CHEK2 & $F G F 2$ & $F G F 10$ & $J A K 2$ & MYCL1 & PTEN & \\
\hline$B R C A 2$ & $E G F R$ & $F G F 3$ & $F G F 14$ & KIT & $M Y C N$ & $R A F 1$ & \\
\hline CCND1 & $E R B B 2$ & $F G F 4$ & $F G F 19$ & KRAS & NRAS & $R E T$ & \\
\hline \multicolumn{8}{|c|}{ Fusions and splice variants (from RNA) } \\
\hline$A B L 1$ & BRCAl & $E R G$ & FGFR1 & $J A K 2$ & MSH2 & NTRK3 & PPARG \\
\hline AKT3 & FRCA2 & ESR1 & $F G F R 2$ & $K D R$ & $M Y C$ & NTRK3 & $R A F 1$ \\
\hline$A L K$ & CDK4 & ETS1 & FGFR3 & $K I F 5 B$ & NOTCH1 & PAX3 & $R E T$ \\
\hline$A R$ & $C S F 1 R$ & ETV1 & FGFR4 & KIT & NOTCH2 & $P A X 7$ & ROS1 \\
\hline$A X L$ & $E G F R$ & ETV4 & FLI1 & $K M T 2 A$ & NOTCH3 & PDGFRA & RPS6KB1 \\
\hline$B C L 2$ & EML4 & ETV5 & FLT1 & $M E T$ & $N R G 1$ & $P D G F R B$ & TMPRSS2 \\
\hline$B R A F$ & $E R B B 2$ & EWSR1 & FLT3 & MLLT3 & NTRK1 & PIK $3 C A$ & \\
\hline
\end{tabular}

The gene panels cover 170 genes for mutational analysis and 59 genes for copy number analysis (Table I). For mutational analysis, fastaq files were uploaded on the BaseSpace Sequence Hub (Illumina) for variant interpretation. Only variants in coding regions and promoter regions or splice variants were retained. In addition, only variants that were present in $<1 \%$ of the population according to Exome Aggregation Consortium (http://exac.broadinstitute.org/) and 1000 Genomes Project data (https://www.internationalgenome.org/) and present in $>5 \%$ of reads with a minimum read depth of 250 were retained. All retained variants were reviewed by reference websites, including Catalogue of Somatic Mutations in Cancer (https://cancer.sange0r.ac.uk/cosmic/), Exome Variant Server (https://evs.gs.washington.edu/EVS/), OncoKB (https://oncokb.org/), and dbSNP (https://www.ncbi.nlm.nih.gov/snp/), and only pathogenic variants were selected. In terms of copy number analysis, genes with more than 2-fold changes relative to the average level were considered amplified. Genes with less than 0.7 -fold changes relative to the average level were considered to undergo a significant loss in copy number. We also performed total nucleic acid extraction to obtain the RNA. RNAs were analyzed for fusion and splice variants using the ArcherDx FusionPlex Assay and Solid Tumor Assay, which together cover 55 genes (ArcherDx, Boulder, CO, USA).

Statistical analysis. A Mann-Whitney U-test was performed to compare differences in continuous variables, such as age, invasion depth, and horizontal spread, between GMC and UEA. A linear-bylinear association test was performed to examine whether significant differences exist in discrete variables between GMC and UEA. Univariate survival analysis was performed to examine the 
prognostic significance of the histological subtype with respect to DFS and OS. A Kaplan-Meier plot was used to display survival curves. All statistical analyses were performed using IBM SPSS Statistics for Windows, version 23.0 (IBM Corp., Armonk, NY, USA). Statistical significance was defined as $p<0.05$.

\section{Results}

Baseline characteristics. Table II summarizes the baseline clinicopathological characteristics of patients with GMC or UEA. The mean ages of eight and 10 patients with GMC and UEA were 44.2 (range $=40-55$ years) and 46.8 years (range $=32-63$ years), respectively. All patients $(8 / 8 ; 100.0 \%)$ with GMC underwent radical hysterectomy. Eight $(80.0 \%)$ and two $(20.0 \%)$ of the 10 UEA patients underwent radical hysterectomy and trachelectomy, respectively. Regarding the immunostaining results, p16 expression was found to be block-positive in all UEA cases $(10 / 10 ; 100.0 \%)$, and either patchy positive $(5 / 8 ; 62.5 \%)$ or negative $(3 / 8 ; 37.5 \%)$ in the GMC cases. Four $(50.0 \%)$ cases of GMC exhibited diffuse and strong nuclear p53 positivity, and the remaining four $(50.0 \%)$ cases demonstrated complete loss of p53 expression. All UEA cases exhibited p53 expression that was patchy and weakly positive. In HPV genotyping analysis, 10 UEAs exhibited high-risk HPV, but in GMC, high-risk HPV was not detected in any of the cases examined. Most (8/10; $80.0 \%$ ) UEA patients were diagnosed as having FIGO stage I tumors, whereas all $(8 / 8 ; 100.0 \%)$ GMCs were staged at II or higher. Three $(37.5 \%)$ and one $(12.5 \%)$ patients were diagnosed with FIGO stage III and IV tumors, respectively. One patient with FIGO stage IA1 disease received no further treatment. Eight of the 18 patients whose tumors were FIGO stage IIB or higher received postoperative concurrent chemoradiation therapy. The remaining nine patients (FIGO stage IA2, two; IB1, two; IB2, three; and IIA, two) underwent postoperative radiation therapy. The mean postoperative follow-up periods were 29.9 and 44.1 months in GMC and UEA patients, respectively. Distant metastases were identified in six $(75.0 \%)$ GMC patients. The metastatic organs included the lungs $(2 / 6)$, ovaries $(1 / 6)$, liver $(1 / 6)$, colon (1/6), and bones (1/6). One patient with Peutz-Jeghers syndrome (case 7) was diagnosed with GMC. She was diagnosed with Peutz-Jeghers syndrome nine years ago and received small bowel resection for ileocolic intussusception due to multiple hamartomatous polyps of the jejunum.

Histopathological features. Representative photomicrographs that depict the histopathological features of GMC and UEA are shown in Figure 1. GMC consisted of mucin-containing epithelium, which invades the cervical stroma (Figure 1A). The tumor cells formed variable-sized, angulated, or cystic glands, with solid regions and infolded papillae (Figure 1B). These glands were irregular and dilated (Figure 1C), and frequently exhibited back-to-back arrangement, cribriform
Table II. Baseline characteristics of gastric-type mucinous carcinoma (GMC) and usual-type endocervical adenocarcinoma (UEA).

\begin{tabular}{|c|c|c|}
\hline \multirow[t]{2}{*}{ Characteristic } & \multicolumn{2}{|c|}{ Number of cases $(\%)$} \\
\hline & GMC $(n=8)$ & UEA $(n=10)$ \\
\hline \multicolumn{3}{|l|}{ Age (years) } \\
\hline Mean \pm Standard deviation & $44.2 \pm 4.7$ & $46.8 \pm 11.3$ \\
\hline Range & $40-55$ & $32-63$ \\
\hline \multicolumn{3}{|l|}{ Invasion depth (mm) } \\
\hline Mean \pm Standard deviation & $17.3 \pm 3.6$ & $6.8 \pm 4.0$ \\
\hline Range & $14-23$ & $2-14$ \\
\hline \multicolumn{3}{|l|}{ Horizontal spread (mm) } \\
\hline Mean \pm Standard deviation & $35.0 \pm 10.4$ & $21.6 \pm 14.9$ \\
\hline Range & $23-54$ & $4-50$ \\
\hline \multicolumn{3}{|l|}{ Type of surgical treatment } \\
\hline Radical hysterectomy & $8(100.0)$ & $8(80.0)$ \\
\hline Radical trachelectomy & $0(0.0)$ & $2(20.0)$ \\
\hline \multicolumn{3}{|l|}{ p16 expression } \\
\hline Block positive & $0(0.0)$ & $10(100.0)$ \\
\hline Patchy positive & $5(62.5)$ & $0(0.0)$ \\
\hline Negative & $3(37.5)$ & $0(0.0)$ \\
\hline \multicolumn{3}{|l|}{ p53 expression } \\
\hline Diffuse, strong positive & $4(50.0)$ & $0(0.0)$ \\
\hline Complete loss & $4(50.0)$ & $0(0.0)$ \\
\hline Patchy positive & $0(0.0)$ & $10(100.0)$ \\
\hline \multicolumn{3}{|l|}{ Human papillomavirus status } \\
\hline High-risk detected & $0(0.0)$ & $10(100.0)$ \\
\hline Not detected & $7(87.5)$ & $0(0.0)$ \\
\hline Not applicable & $1(12.5)$ & $0(0.0)$ \\
\hline \multicolumn{3}{|l|}{ Initial stage } \\
\hline I & $0(0.0)$ & $8(80.0)$ \\
\hline II & $4(50.0)$ & $0(0.0)$ \\
\hline III & $3(37.5)$ & $2(20.0)$ \\
\hline IV & $1(12.5)$ & $0(0.0)$ \\
\hline \multicolumn{3}{|l|}{ Local recurrence } \\
\hline Present & $1(12.5)$ & $2(20.0)$ \\
\hline Absent & $7(87.5)$ & $8(80.0)$ \\
\hline \multicolumn{3}{|l|}{ Distant metastasis } \\
\hline Present & $6(75.0)$ & $1(10.0)$ \\
\hline Absent & $2(25.0)$ & $9(90.0)$ \\
\hline \multicolumn{3}{|l|}{ Follow-up period (month) } \\
\hline Mean \pm Standard deviation & 29.9 & 44.1 \\
\hline Range & $12-41$ & $30-51$ \\
\hline
\end{tabular}

structure, and solid architecture. Fragmented glands and individually scattered tumor cells were also noted (Figure 1D). The surrounding stroma demonstrated evident desmoplasia (Figure 1E), although some foci featured peritumoral stroma that lacked a desmoplastic response. The glands, from which the mucin protein is spilled, were lined by malignant-appearing mucinous epithelium. Inflammatory infiltrates and fibrosis were associated with one another. The tumor cells abundantly possessed clear (Figure 1F) or pale eosinophilic (Figure 1G) cytoplasm with a foamy appearance and relatively distinct cell borders. The tumor cell nuclei were enlarged and pleomorphic; furthermore, these nuclei 

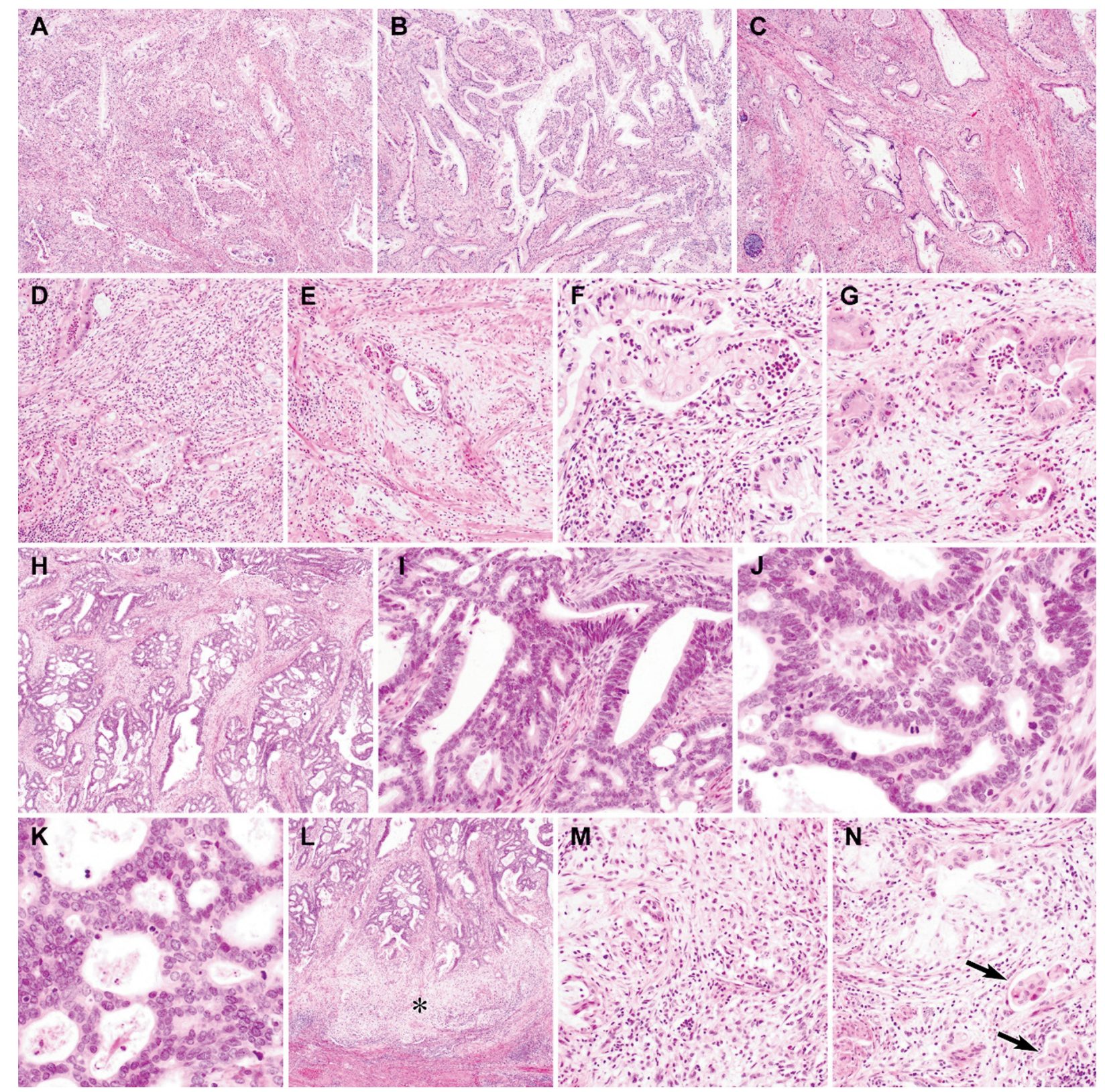

Figure 1. Histopathological features of gastric-type mucinous carcinoma (GMC; A to G) and usual-type endocervical adenocarcinoma (UEA; $H$ to $N)$. A: GMC consists of variable-sized, irregular-shaped glands that are lined by mucin-containing epithelium and infiltrate the cervical stroma. B: The tumor cells form angulated or complicated glands that anastomose each other. C: These glands invade haphazardly the deep cervical stroma and are associated with stromal desmoplasia. D: The fragmented glands and individually scattered tumor cells are admixed with extensive lymphoplasmacytic and eosinophilic stromal infiltrates. E: The surrounding stroma demonstrates the fibromyxoid desmoplastic reaction. F: The tumor cells possess abundant clear cytoplasm with a foamy appearance and relatively distinct cell borders. G: The tumor cell nuclei are enlarged and pleomorphic and exhibit coarse chromatin, variable-sized nucleoli, and membrane irregularity. H: UEA displays a complex glandular architecture, consisting mainly of cribriform glands and some dilated glandular lumina. I: The tumor cells demonstrate characteristic pseudostratified columnar epithelium with elongated, hyperchromatic nuclei. J: The tumor cell nuclei possess conspicuous nucleoli. Mitotic figures were frequently identified, especially in the apical zone of the amphophilic to eosinophilic cytoplasm. K: Apoptotic bodies and karyorrhectic debris are readily visible within the epithelium and glandular spaces. L: In a single case of UEA, the microcystic, elongated, and fragmented (MELF) patterns of invasion are noted. At the invasive tumor front $\left(^{*}\right)$, the tumor cells or glands were obscured by striking stromal desmoplasia and myxoinflammatory responses. Thus, they are not apparent at low-power magnification. M: The tumor cells demonstrate eosinophilic or vacuolated cytoplasm and are surrounded by a fibromyxoid or edematous stroma that is associated with inflammatory cells. N: In some foci, the tumor cell clusters exhibit epithelioid morphology, resembling histiocytes that are aggregated within lymphatic spaces (black arrows). A to $N$, hematoxylin and eosin staining. Original magnification, $A$ to $C, \times 40 ; D$ and $E, \times 100 ; F$ and $G, \times 200 ; H, \times 40 ; I, \times 200 ; J$ and $K, \times 400 ; L, \times 40 ; M$ and $N \times 200$. 
exhibited coarse chromatin, variable-sized nucleoli, and membrane irregularity. Mitotic and apoptotic activities were low, and atypical mitotic figures were not identified. In cases with parametrial extension, the neoplastic glands infiltrated through the entire cervical stroma into the parametrial fibroadipose tissue. The glands and associated stromal desmoplasia were identified between thick-walled blood vessels. In three of the six metastatic tumors, several foci of signet ring cell differentiation (2/6) and dedifferentiation with sarcomatoid morphology (1/6) were observed.

UEA displayed complex architectural patterns (Figure 1H), which consisted of round-to-oval shape, mucin-poor glands, microcysts, and cribriform structures. The dilated glandular lumina contained necrotic debris. The tumor cells exhibited a characteristic pseudostratified columnar epithelium with enlarged, elongated, and hyperchromatic nuclei (Figure 1I). The tumor nuclei possessed conspicuous nucleoli. Mitotic figures were frequently identified, especially in the apical zone of the amphophilic-to-eosinophilic cytoplasm (Figure 1J). Apoptotic bodies and karyorrhectic debris were readily visible within the epithelium and glandular spaces (Figure $1 \mathrm{~K}$ ). Although most areas exhibited relative mucin depletion and pseudostratified nuclei, mucin pools and a single layer of the columnar epithelium with appreciable intracytoplasmic mucin were noted.

In a single case of UEA, microcystic, elongated, and fragmented (MELF) patterns of invasion were noted. At the invasive tumor front, the tumor glands displayed a microcystic and elongated appearance and were lined by flattened epithelium. Since the tumor cells or glands were obscured by striking stromal desmoplasia and the myxoinflammatory response, they were not apparent at low-power magnification (Figure 1L). The tumor cells exhibited eosinophilic or vacuolated cytoplasm and were surrounded by a fibromyxoid or edematous stroma that was associated with inflammatory cells (Figure 1M). In some foci, the tumor cell clusters exhibited epithelioid morphology, resembling histiocytes that were aggregated within the lymphatic spaces (Figure 1N).

In case 7 , lobular endocervical glandular hyperplasia (LEGH) was associated with GMC. Distinctly lobular architecture was noted with proliferated small round glands that surround a central cystically dilated duct (Figure 2A). The lesion was confined to the inner half of the cervical wall and located within the upper endocervix (Figure 2B). Each gland was lined by mucin-containing columnar cells (Figure 2C), and possesses basally located, uniform, and small nuclei (Figure 2D). No nuclear atypia was found. The intervening stroma was unremarkable (Figure 2E), without desmoplasia or significant inflammation.

Representative photomicrographs that depict immunoreactivities for p53 and p16 in GMC and UEA are shown in Figure 3. In GMCs (Figure 3A), p16 expression was patchy positive $(5 / 8)$ or negative (3/8; Figure $3 \mathrm{~B})$. All GMCs expressed a mutant p53 expression pattern. Three of the eight GMCs displayed completely negative for p53 immunostaining (nonsense of frameshift mutation pattern; Figure 3C), and the remaining four cases showed uniform and strong p53 expression in nearly all the tumor cell nuclei (missense mutation pattern; Figure 3D). In contrast, all UEAs (Figure 3E) demonstrated diffuse and strong nuclear immunoreactivity for p16 (block p16 positivity; Figure 3F) and patchy and weak-to-moderate nuclear immunoreactivity for p53 (wild-type p53 expression pattern; Figure 3G).

Clinicopathological differences. Table III summarizes the clinicopathological differences between GMC and UEA. GMC exhibited significantly deeper invasion depth (mean: $17.3 \mathrm{~mm}$ versus $6.8 \mathrm{~mm} ; p<0.001)$, greater horizontal spread (mean: $35.0 \mathrm{~mm}$ versus $21.6 \mathrm{~mm} ; p=0.039$ ), more advanced FIGO staging ( $p=0.001$ ), and more frequent distant metastasis $(p=0.013)$ than UEA. Additionally, parametrial extension $(p=0.015)$, parametrial resection margin involvement $(p=0.023)$, vaginal extension $(p=0.043)$, and vaginal resection margin involvement $(p=0.023)$ were more frequently observed in GMC than in UEA. All of the UEA cases exhibited negative resection margins in the vagina or parametrium, whereas half of the GMCs involved either vaginal or parametrial resection margins. No statistically significant differences were found in lymphovascular invasion, perineural invasion, adnexal extension, peritoneal metastases, and lymph node metastasis between GMC and UEA.

Differences in patient outcome. Kaplan-Meier plots for DFS and OS are shown in Figure 4. During the follow-up period, one $(12.5 \%)$ and six $(75.0 \%)$ GMC patients developed recurrence and metastasis, respectively. The DFS rate for GMC patients was significantly lower than that for UEA patients ( $p=0.032$; Figure 4A). The median DFS time for patients with GMC was 19 months, compared to more than four years for UEA patients. Patients with UEA had stable DFS rates of $90 \%$ between one and two years after surgery and $80.0 \%$ after two and a half years. In contrast, GMC patients displayed a steady decline in the DFS rate during the first two years after surgery. DFS rates for GMC patients were $62.5 \%$ for one year and $25.0 \%$ for two years. In addition, the OS time for GMC patients was shorter than that of patients with UEA, although the difference in the OS rate between the two groups was not statistically significant ( $p=0.076$; Figure 4B). The OS rate for UEA patients was over $90.0 \%$ during the observation period (51 months), whereas the OS rate for GMC patients at the last follow-up was only $36.5 \%$.

Targeted sequencing results. Table IV and Figure 5 summarize the pathogenic mutations that were detected in GMC and UEA tissue samples. All except one patient harbored at least one pathogenic mutation. Two GMCs (case 1 and 2) that harbored 

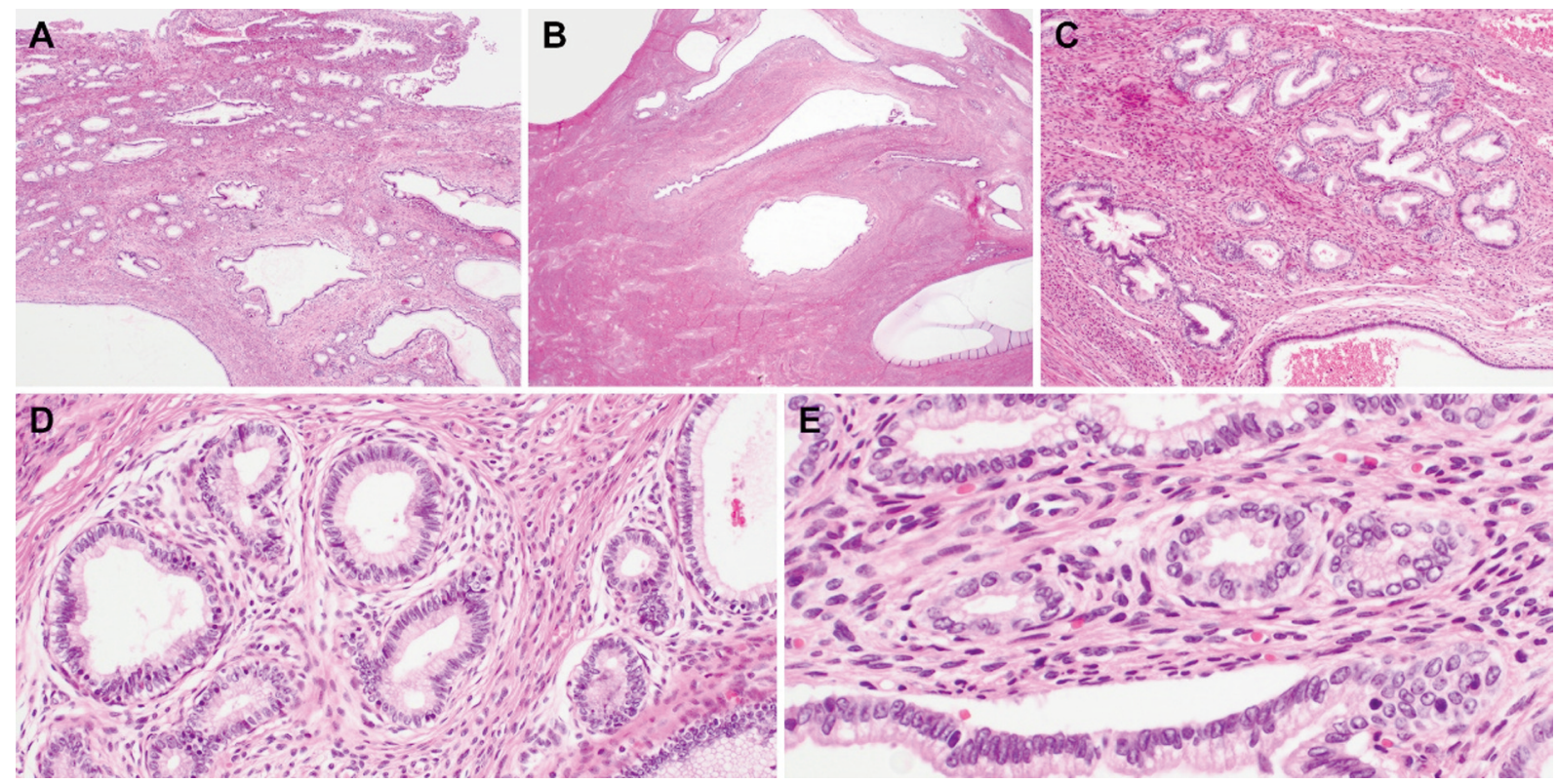

Figure 2. Histopathological features of lobular endocervical glandular hyperplasia. A: A distinctly lobular architecture consists of small round glands and cystically dilated ducts. B: The lesion is confined to the inner half of the cervical wall and.C: Each gland is lined by mucin-containing columnar epithelium. D: The epithelial cells possess basally located, uniform nuclei. Each cell does not exhibit any nuclear pleomorphism. E: The intervening stroma displays no inflammation or desmoplasia. A to E, hematoxylin and eosin staining. Original magnification, A, $\times 12.5 ; B, \times 40 ; C$, $\times 100 ; D, \times 200 ; E, \times 400$.
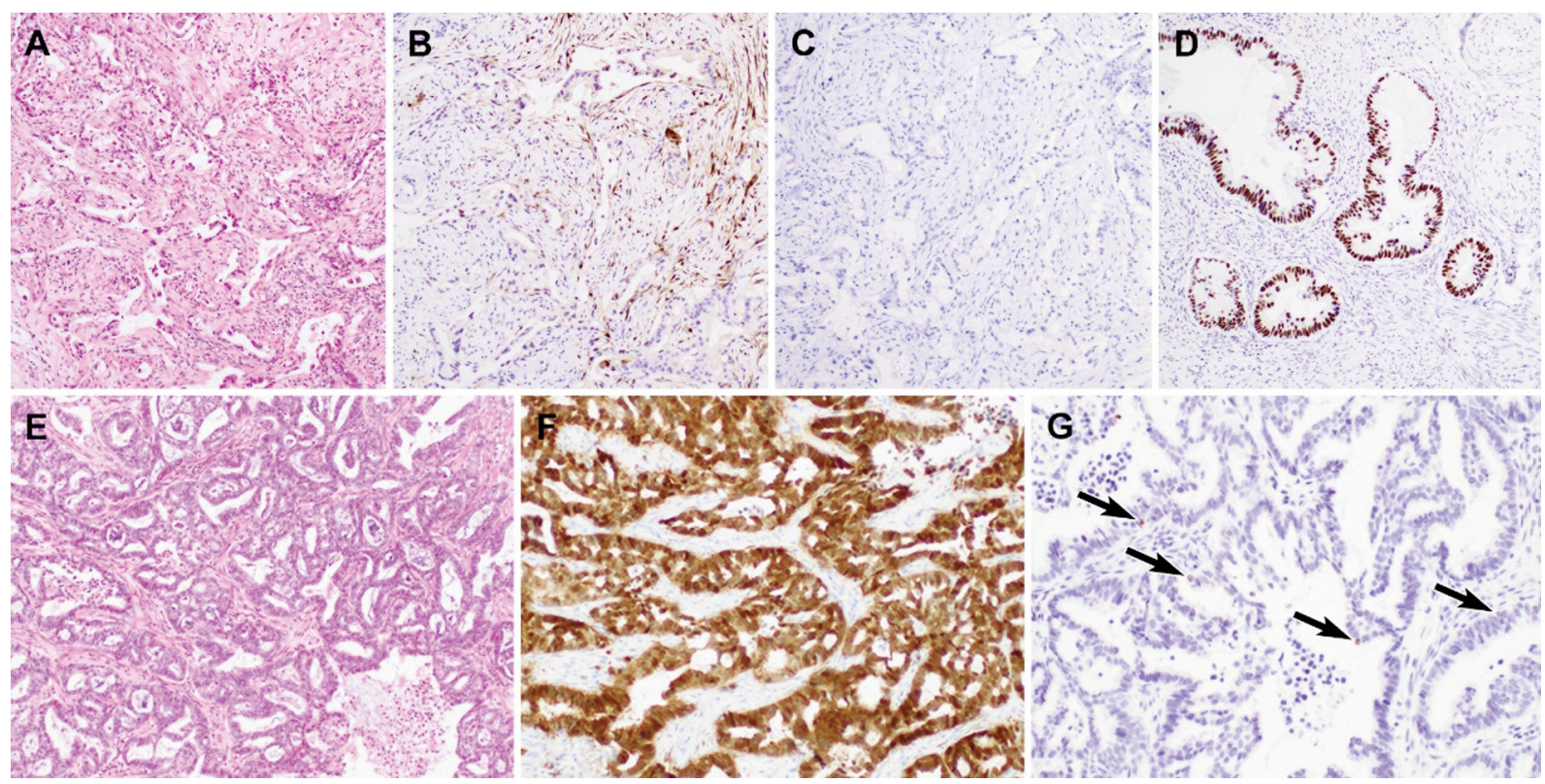

Figure 3. Immunostaining results of gastric-type mucinous carcinoma (GMC; A to D) and usual-type endocervical adenocarcinoma (UEA; E to G). (black arrows). A: GMC. B: The GMC cells do not express p16, indicating that this tumor is independent of the human papillomavirus (HPV) infection. Instead, the spindle- or stellate-shaped stromal cells exhibit strong nuclear and cytoplasmic immunoreactivity for p16. C and D: p53 immunostaining reveals that the GMC cells display complete loss $(C)$ or diffuse and strong expression $(D)$, indicating the presence of the tumor protein 53 (TP53) mutation. E: UEA. F: The UEA cells demonstrate diffuse and strong nuclear p16 expression (block p16 positivity), indicating that UEA is associated with high-risk HPV infection. G: In contrast to p16 expression, p53 expression is weak and patchy in scattered tumor cells, indicating wild-type TP53. A and E, hematoxylin and eosin staining; $B$ to $D, F$, and $G$, polymer method. Original magnification, A to D, $\times 100 ; E$, $\times 100 ; F$ and $G, \times 200$. 
missense TP53 (tumor protein 53) mutations [c.724T>A (p.Cys242Ser) and c.818G $>\mathrm{A}$ (pArg273His)], which were compatible with diffuse and strong nuclear p53 immunoreactivity (missense mutation pattern of p53 expression). Each of the two cases exhibited an additional mutation, including the missense KRAS (Kirsten rat sarcoma viral oncogene homolog) mutation [c.35G $>$ A (p.Gly12Asp)] and frameshift deletion of the NFI (neurofibromin 1) gene [c.3037delA (p.Thr1013Argfs)]. The latter mutation was also detected in another case of GMC (case 3). Three cases of GMC harbored nonsense (case 4) and missense (case 5) mutations and frameshift insertion (case 7) of the CDKN2A (cyclin-dependent kinase inhibitor 2A) gene [c.172C $>\mathrm{T}$ (p.Arg58Ter), c.224C $>\mathrm{T}$ (p.Pro75Leu), and c.213_214insT (p.Arg71fs), respectively]. In addition, a patient with Peutz-Jeghers syndrome (case 7) was identified to foster the STK11 (serine/threonine kinase 11) mutation [c.470T $>\mathrm{G}$ (p.Phe157Cys)], along with the missense KRAS mutation [c.176C $>\mathrm{G}$ (p.Ala59Gly)] and nonsense ARIDIA (AT-rich interaction domain 1A) mutation [c.802C $>\mathrm{T}$ (p.Gln268Ter)]. In a single case of GMC (case 6), MDM2 (mouse double minute 2 homolog) amplification was detected.

Half $(5 / 10)$ of the UEA cases harbored at least one pathogenic mutation. The HRAS (Harvey rat sarcoma viral oncogene homolog) missense mutation [c.34G $>$ T (p.Gly12Cys)] was detected in one case (case 1). This case also exhibited the TP53 splice site mutation (c.376-2A>T), which was identified in another case (case 2). Two cases (case 3 and 4) harbored the PIK3CA (phosphatidylinositol4,5-bisphosphate 3-kinase catalytic subunit alpha) missense mutation [c.1633G $>$ A (p.Glu545Lys)]. The BRCA2 (breast cancer 2, early onset) nonsense mutations [c.8951C $>A$ (p.Ser2984Ter) and c.1850C >A (p.Ser617Ter)] were identified in two cases, one of which harbored the PIK3CA mutation (case 4), and the other (case 5) of which harbored the BRCA2 mutation only.

\section{Discussion}

We observed that GMC, compared to UEA, was significantly associated with larger horizontal spread, deeper invasion depth, parametrial extension, vaginal extension, more advanced staging, and distant metastasis. These findings clearly confirm the notion that GMC is diagnosed at advanced stages and displays more aggressive behavior than UEA (7). GMC frequently metastasizes to the extrapelvic organs and exhibits worse prognosis (24). Our results are consistent with those of a recent study by Nishio et al. (25) with the largest GMC cohort, which ultimately demonstrated that GMC is more significantly associated with bulky mass, deep stromal invasion, and parametrial invasion than UEA. Our observations that all GMC cases classified as FIGO stage II-IV, but the majority $(80.0 \%)$ of the UEA patients had FIGO stage I tumors is in accordance with those of
Table III. Clinicopathological differences between gastric-type mucinous carcinoma (GMC) and usual-type endocervical adenocarcinoma (UEA).

\begin{tabular}{|c|c|c|c|}
\hline \multirow[t]{2}{*}{ Characteristic } & \multicolumn{2}{|c|}{ Number of cases $(\%)$} & \multirow[t]{2}{*}{$p$-Value } \\
\hline & GMC & UEA & \\
\hline Age & $44.2 \pm 4.7$ & $46.8 \pm 11.3$ & 0.542 \\
\hline Invasion depth (mm) & $17.3 \pm 3.6$ & $6.8 \pm 4.0$ & $<0.001 *$ \\
\hline Horizontal spread (mm) & $35.0 \pm 10.4$ & $21.6 \pm 14.9$ & $0.039 *$ \\
\hline \multicolumn{4}{|l|}{ Parametrial extension } \\
\hline Present & 7 (87.6) & $2(20.0)$ & $0.015^{*}$ \\
\hline Absent & $1(12.5)$ & $8(80.0)$ & \\
\hline \multicolumn{4}{|l|}{$\begin{array}{c}\text { Parametrial resection } \\
\text { margin involvement }\end{array}$} \\
\hline Present & $4(50.0)$ & $0(0.0)$ & $0.023^{*}$ \\
\hline Absent & $4(50.0)$ & $10(100.0)$ & \\
\hline \multicolumn{4}{|l|}{$\begin{array}{l}\text { Endomyometrial } \\
\text { extension }\end{array}$} \\
\hline Present & $4(50.0)$ & $4(40.0)$ & 1.000 \\
\hline Absent & $4(50.0)$ & $4(40.0)$ & \\
\hline Not applicable & $0(0.0)$ & $2(20.0)$ & \\
\hline \multicolumn{4}{|l|}{ Vaginal extension } \\
\hline Present & $5(62.5)$ & $1(10.0)$ & $0.043^{*}$ \\
\hline Absent & $3(37.5)$ & $9(90.0)$ & \\
\hline \multicolumn{4}{|l|}{$\begin{array}{l}\text { Vaginal resection } \\
\text { margin involvement }\end{array}$} \\
\hline Present & $4(50.0)$ & $0(0.0)$ & $0.023^{*}$ \\
\hline Absent & $4(50.0)$ & $10(100.0)$ & \\
\hline \multicolumn{4}{|l|}{$\begin{array}{l}\text { Lymphovascular } \\
\text { invasion }\end{array}$} \\
\hline Present & $3(37.5)$ & $4(40.0)$ & 1.000 \\
\hline Absent & $5(62.5)$ & $6(60.0)$ & \\
\hline \multicolumn{4}{|l|}{ Perineural invasion } \\
\hline Present & $3(37.5)$ & $1(10.0)$ & 0.275 \\
\hline Absent & $5(62.5)$ & $9(90.0)$ & \\
\hline \multicolumn{4}{|l|}{ Adnexal extension } \\
\hline Present & $1(12.5)$ & $2(20.0)$ & 1.000 \\
\hline Absent & $7(87.5)$ & $8(80.0)$ & \\
\hline \multicolumn{4}{|l|}{$\begin{array}{l}\text { Pelvic peritoneal } \\
\text { metastasis }\end{array}$} \\
\hline Present & $1(12.5)$ & $0(0.0)$ & 0.444 \\
\hline Absent & $7(87.5)$ & $10(100.0)$ & \\
\hline \multicolumn{4}{|l|}{$\begin{array}{l}\text { Extrapelvic peritoneal } \\
\text { metastasis }\end{array}$} \\
\hline Present & $1(12.5)$ & $0(0.0)$ & 0.444 \\
\hline Absent & $7(87.5)$ & $10(100.0)$ & \\
\hline \multicolumn{4}{|l|}{$\begin{array}{l}\text { Lymph node } \\
\text { metastasis }\end{array}$} \\
\hline Present & $4(50.0)$ & $2(20.0)$ & 0.321 \\
\hline Absent & $4(50.0)$ & $8(80.0)$ & \\
\hline \multicolumn{4}{|l|}{ Initial stage } \\
\hline I & $0(0.0)$ & $8(80.0)$ & $0.001 *$ \\
\hline II-IV & $8(100.0)$ & $2(20.0)$ & \\
\hline \multicolumn{4}{|l|}{ Local recurrence } \\
\hline Present & $1(12.5)$ & $2(20.0)$ & 1.000 \\
\hline Absent & $7(87.5)$ & $8(80.0)$ & \\
\hline \multicolumn{4}{|l|}{ Distant metastasis } \\
\hline Present & $6(75.0)$ & $1(10.0)$ & $0.013^{*}$ \\
\hline Absent & $2(25.0)$ & $9(90.0)$ & \\
\hline
\end{tabular}

*Statistically significant. 
A

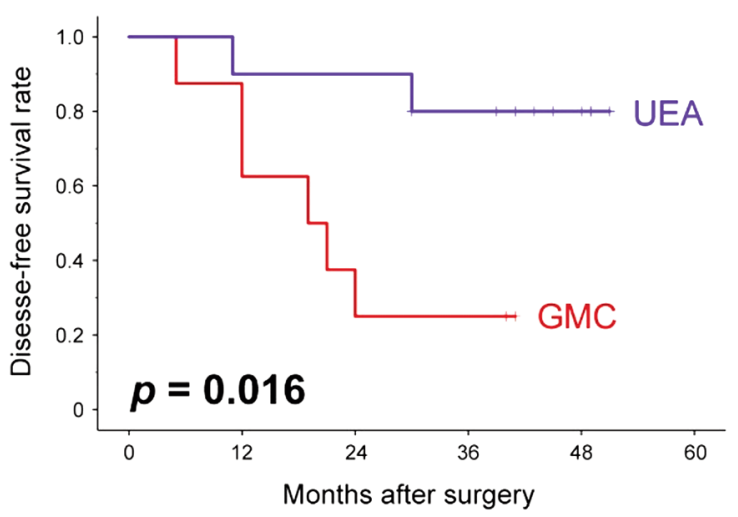

B

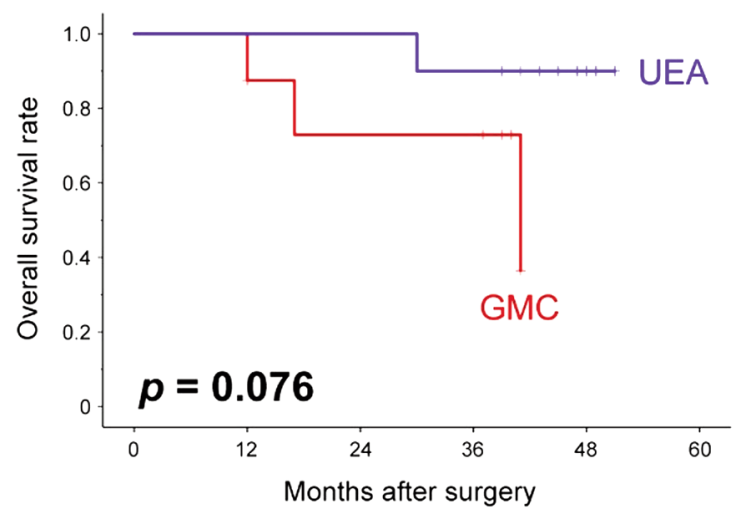

Figure 4. Kaplan-Meier plots for disease-free survival (DFS) (A) and overall survival (OS) (B) of patients with gastric-type mucinous carcinoma (GMS) or usual-type endocervical adenocarcinoma (UEA). The survival rates are indicated in red for GMC and in blue for UEA. A: DFS rate for GMC patients is significantly lower than that for UEA. B: OS time for GMC patients is also shorter than that of patients with UEA, although the difference in the OS rate between the two groups was not statistically significant.

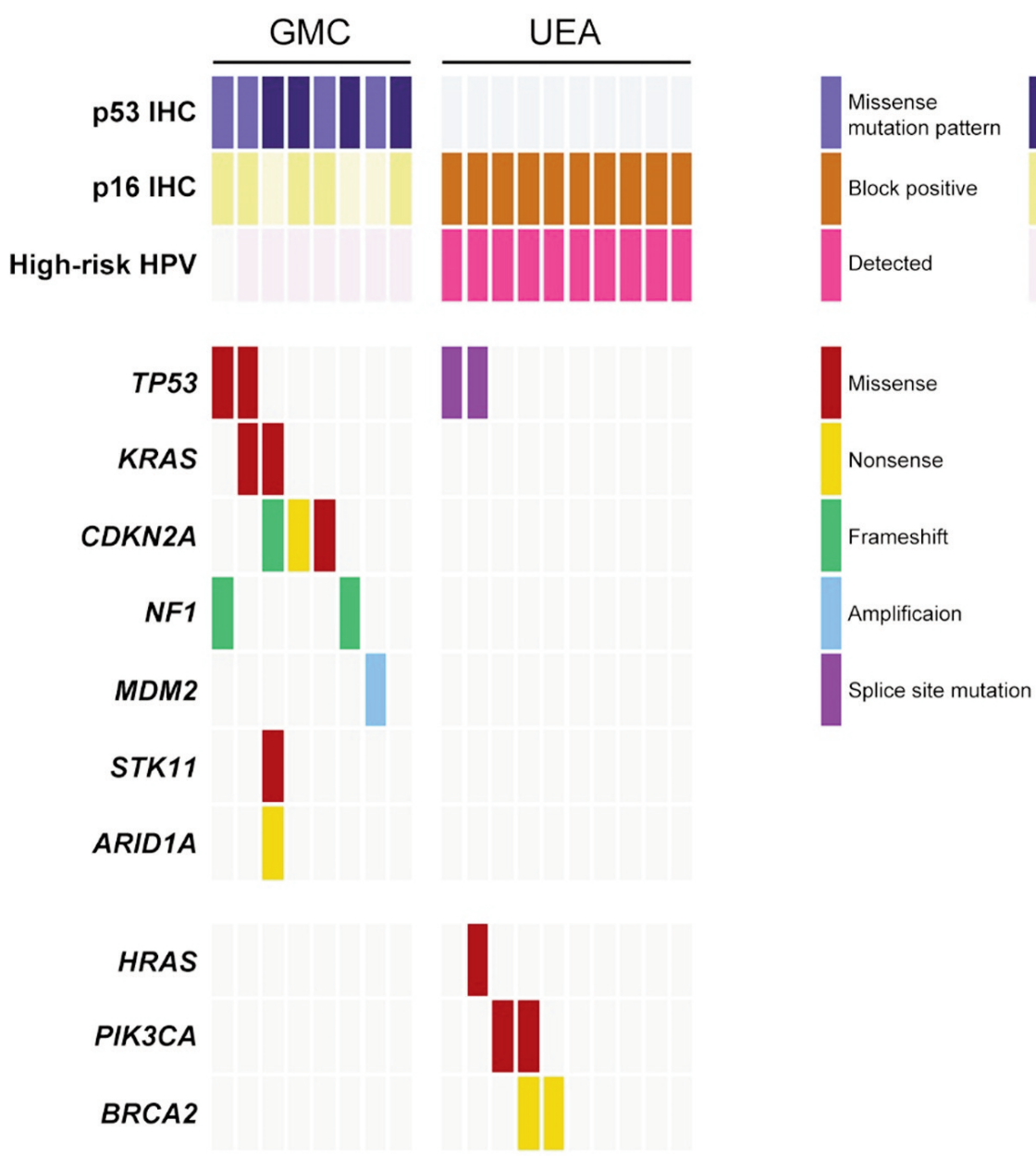

Figure 5. Targeted sequencing results, immunophenotype, and human papillomavirus (HPV) status of gastric-type mucinous carcinoma (GMC) and usual-type endocervical adenocarcinoma (UEA). 
Table IV. Pathogenic mutations in gastric-type mucinous carcinoma (GMC) and usual-type endocervical adenocarcinoma (UEA).

\begin{tabular}{|c|c|c|c|c|}
\hline Case No & Gene & Mutation type & Sequence change & Predicted effect \\
\hline \multicolumn{5}{|l|}{ GMC } \\
\hline \multirow[t]{2}{*}{1} & $K R A S$ & Missense & c. $35 \mathrm{G}>\mathrm{A}$ & p.Gly12Asp \\
\hline & TP53 & Missense & c. $724 \mathrm{~T}>\mathrm{A}$ & p.Cys242Ser \\
\hline \multirow[t]{2}{*}{2} & TP53 & Missense & c. $818 \mathrm{G}>\mathrm{A}$ & pArg273His \\
\hline & $N F 1$ & Frameshift & c.3037delA & p.Thr1013Argfs \\
\hline 3 & $N F 1$ & Frameshift & c.3037delA & p.Thr1013Argfs \\
\hline 4 & $C D K N 2 A$ & Nonsense & c. $172 \mathrm{C}>\mathrm{T}$ & p.Arg58Ter \\
\hline 5 & $C D K N 2 A$ & Missense & c. $224 \mathrm{C}>\mathrm{T}$ & p.Pro75Leu \\
\hline 6 & $M D M 2$ & Copy number variation & Amplification & Overexpression \\
\hline \multirow[t]{4}{*}{7} & KRAS & Missense & c. $176 \mathrm{C}>\mathrm{G}$ & p.Ala59Gly \\
\hline & STK11 & Missense & c. $470 \mathrm{~T}>\mathrm{G}$ & p.Phe157Cys \\
\hline & $C D K N 2 A$ & Frameshift & c.213_214insT & p.Arg71fs \\
\hline & ARID1A & Nonsense & c. $802 \mathrm{C}>\mathrm{T}$ & p.Gln268Ter \\
\hline 8 & Not detected & & & \\
\hline \multicolumn{5}{|l|}{ UEA } \\
\hline \multirow[t]{2}{*}{1} & $H R A S$ & Missense & c. $.34 \mathrm{G}>\mathrm{T}$ & p.Gly12Cys \\
\hline & TP53 & Splice acceptor variant & c. $376-2 \mathrm{~A}>\mathrm{T}$ & \\
\hline 2 & TP53 & Splice acceptor variant & c. $376-2 \mathrm{~A}>\mathrm{T}$ & \\
\hline 3 & PIK3CA & Missense & c. $1633 \mathrm{G}>\mathrm{A}$ & p.Glu545Lys \\
\hline \multirow[t]{2}{*}{4} & $P I K 3 C A$ & Missense & c. $1633 \mathrm{G}>\mathrm{A}$ & p.Glu545Lys \\
\hline & $B R C A 2$ & Nonsense & c. $8951 \mathrm{C}>\mathrm{A}$ & p.Ser2984Ter \\
\hline 5 & BRCA2 & Nonsense & c. $1850 \mathrm{C}>\mathrm{A}$ & p.Ser617Ter \\
\hline 6 & Not detected & & & \\
\hline 7 & Not detected & & & \\
\hline 8 & Not detected & & & \\
\hline 9 & Not detected & & & \\
\hline 10 & Not detected & & & \\
\hline
\end{tabular}

KRAS: Kirsten rat sarcoma viral oncogene homolog, TP53: tumor protein 5, NF1: neurofibromin 1, CDKN2A: cyclin-dependent kinase inhibitor 2A, MDM2: mouse double minute 2 homolog, STK11: serine/threonine kinase 11, ARID1A: AT-rich interaction domain 1A, HRAS: Harvey rat sarcoma viral oncogene homolog, PIK3CA: phosphatidylinositol-4,5-bisphosphate 3-kinase catalytic subunit alpha, BRCA2: breast cancer 2, early onset.

Karamurzin et al. (24), which showed that GMC presents at more advanced stages than UEA. Nishio et al. (25) reported that lymphovascular invasion, ovarian metastasis, pelvic lymph node metastasis, and recurrence, all of which are conventional pathological features that predict worse prognosis, were more frequently observed in GMC compared to in UEA. However, a significant difference was not observed in the frequency of the lymphovascular invasion, adnexal extension, lymph node metastasis, and local recurrence between GMC and UEA. This discrepancy may be attributed to the small number of cases in this study. Nevertheless, we noted that GMC patients developed more frequently metastases to distant organs, including the lungs, liver, ovaries, and colon, than those with UEA. In addition, we found that the surgical resection margin involvement in the vagina and parametrium - which is an important risk factor for postoperative recurrence $(26,27)$ - was more frequent in GMC than in UEA, thus suggesting that additional treatments should be considered to improve local control, such as radiation boosting, in GMC patients.

Despite a small number of cases, we demonstrated that the DFS rate for GMC patients was significantly lower than that for UEA patients. The median DFS time for GMC was 19 months, compared to more than four years for UEA. GMC patients displayed a steady decline in the DFS rate up to $25.0 \%$ during the first two years after surgery, whereas UEA patients exhibited stable DFS rates of $80.0 \%$ or higher for two and a half years after surgery. The OS time for GMC patients was also shorter than that of patients with UEA, although the difference between the two groups was not statistically significant. Our results are consistent with those of previous studies. Kojima et al. (28) found that GMC patients (30\%) had a significantly lowered five-year DFS rate compared to the non-gastric type (77\%), and gastric-type morphology exhibited a significant risk for disease recurrence with a hazard ratio of 4.5. Nishio et al. (25) documented that both DFS and OS were 
worse in patients with GMC than in those with UEA. Karamurzin et al. (24) also reported that GMC exhibited significantly worse disease-specific survival than UEA. Similarly, Park et al. (29) showed that GMC had a significantly shorter interval between surgery and disease recurrence (time to recurrence) compared to UEA. Overall, GMC exhibits aggressive behavior with significantly reduced survival compared to UEA, and GMC is thus considered a distinct entity from UEA.

In this study, we identified mutually exclusive expression of p16 and p53 in UEA and GMC. In all 10 cases of UEA with p16 block-positivity, p53 immunoreactivity was focal and weak, indicating wild-type TP53. In contrast, all eight cases of GMC displayed mutant p53 immunostaining patterns (either diffuse and strongly positive or complete absence) and non-block-type (patchy) p16 expression. These results are in accordance with the HPV-negative status of GMC and the findings of the recent study by Garg et al. (9), which show that all 14 GMCs demonstrated non-block p16 immunoreactivity - consisting of 12 cases with negative expression, one case with focal staining, and one case with patchy expression. Our findings suggest that immunostaining for p16 and p53 allows distinguishing between UEA and GMC. However, in contrast to our results, diffuse and strong nuclear p16 expression has been reported in a rare subset of GMC despite negative molecular testing for HPV (30-32). Moreover, not all GMCs display mutant p53 immunostaining patterns. Garg et al. (9) reported that aberrant p53 expression was observed in nine of 14 GMCs. Similarly, in a previous study by Carleton et al. (30), which featured immunohistochemical analyses on cervical and vaginal GMCs, p53 exhibited wildtype and mutant immunostaining patterns in 27 (58.7\%) and 19 (41.3\%) of the 46 total cases, respectively. Further, two of four GMCs that were reported to exhibit p16 block positivity were negative for HPV. These findings indicate that p16 immunoreactivity in endocervical adenocarcinomas are not necessarily indicative of high-risk HPV-associated tumors, and that p53 expression patterns do not serve as an absolute diagnostic marker to diagnose GMC.

The molecular characteristics of GMC have recently been analyzed using the next-generation sequencing (NGS) technique (33). In the 14 GMCs that were examined, variants were detected most frequently in the TP53, MSH6, CDKN2A, CDKN2B, POLE, SLX4, ARID1A, STK11, BRCA2, and MSH2 genes. Mutations in genes that are associated with one or many of the following pathways were also identified: homologous recombination deficiency, mismatch repair, cell cycle, Fanconi anemia pathway, Notch signaling, and phosphotidylinositol 3kinase/Akt signaling. In accordance with these data, we observed GMCs with mutations in the TP53, CDKN2A, ARIDIA, and STK11 genes. Garg et al. (9) found that GMC resembles endometrial cancer more closely than UEA, in terms of molecular features, since it harbors mutations in the KRAS,
MSH2, MSH6, and POLE genes-mutations that are found in endometrial cancer, but not in UEA.

We noted a MDM2 amplification in a single case of GMC. This is consistent with the study by Garg et al. (9), which identified the same copy number abnormality in two GMC cases. Interestingly, although these two cases were reported to exhibit wild-type p53 immunostaining patterns, our cases suggest that MDM2 amplification demonstrated diffuse and strong p53 expression (missense mutation pattern) without TP53 mutation. MDM2 is a negative regulatory factor for $\mathrm{p} 53$. MDM2 ubiquitinate degrades p53 protein through close-loop negative feedback regulation of MDM2-p53, thereby reducing p53 protein levels (34). Several studies confirm that MDM2 amplification down-regulates p53 expression, and that inhibition of $M D M 2$ expression induces p53 up-regulation (34, 35). Based on the well-known functions of $M D M 2$, our observation of concurrent $M D M 2$ amplification and missense mutation pattern of p53 expression is an uncommon observation. Nevertheless, complex interactions of intracellular signaling pathways that activate p53-related pathways and stabilize the p53 protein are apparent, and our results suggest the possibility of $M D M 2$-independent regulatory mechanisms that up-regulate $\mathrm{p} 53$ protein expression.

We observed splice-site mutations of the TP53 gene in two cases of UEA. No study has found splice-site mutations of TP53 in UEA. The TP53 splice-site mutation (c.376$2 \mathrm{~A}>\mathrm{T}$ ) that we observed had been observed in ovarian cancer and Li-Fraumeni syndrome (36). Although the splice-site mutations of TP53 are very scarce in UEA and their effects have not been well characterized, the possibility that the novel splice-site mutation in this study is involved in the pathogenesis of UEA cannot be excluded. In addition, tuboovarian high-grade carcinoma with TP53 splice-site mutations (c.920-6_922delTCCTAGCAC and c.920-2delA) was found to exhibit wild-type expression patterns in the p53 immunostaining results (18). This study showed patchy and weak p53 expression in two cases of UEA with TP53 splicesite mutations. Although it is known that the p53 immunostaining pattern and TP53 mutational status are correlated in more than $95 \%$ of tubo-ovarian high-grade carcinoma, false-negative staining can occur in a small subset of cases. Since no previous studies have compared p53 expression status and TP53 mutational status in UEA, their correlation cannot be determined. However, we suspect that the discordance between the novel splice-site mutation of the TP53 gene and wild-type p53 immunostaining pattern that was observed in this study is rare.

Peutz-Jeghers syndrome is an autosomal-dominant disorder that carries a mutation in the STK11 gene, which is a tumor suppressor gene that is located in chromosome 19p13.3 (9). Approximately 14\% of GMCs were reportedly associated with Peutz-Jeghers syndrome (37). In this study, we presented a case of GMC that was associated with the 
STK11 mutation and LEGH in a 39-year-old patient with Peutz-Jeghers syndrome who received segmental resection of the small bowel for multiple gastric and jejunal hamartomatous polyps and ileocolic intussusception. LEGH is usually detected incidentally in a hysterectomy or cervical conization specimen. Patients presented watery or mucoid vaginal discharge, which suggests minimal deviation adenocarcinoma (MDA) of the cervix. In this situation, imaging findings may also raise concerns for MDA or GMC, since LEGH appears as a multicystic lesion that occupies the cervix either partially or entirely. Histologically, distinguishing LEGH from MDA is difficult. Architectural pattern at low-power magnification is critical and best observed in a resected specimen. LEGH exhibits the orderly lobular arrangement of mucinous glands that are usually confined to the inner half of the cervical wall, whereas MDA displays a haphazard glandular arrangement of various sizes and shapes that widely infiltrate the stroma, usually with a focal desmoplastic response and nuclear atypia $(24,25)$. Lymphovascular or perineural invasion and parametrial extension, if present, assist in establishing a diagnosis of malignancy (36). In addition, we identified the STK11 mutation [c.470T $>\mathrm{G}$ (p.Phe157Cys)] and KRAS mutation [c.176C $>\mathrm{G}$ (p.Ala59Gly)] from the GMC tissue. This finding of the co-existence of the KRAS mutation [c.187G $>\mathrm{A}$ (p.Glu63Lys)] and STK11 truncation variant [c.396C $>A$ (p.Cys132Ter)] in one case of GMC is consistent with a previous study by Garg et al. (9). Additionally, Matsubara et al. (38) found mutations in one of the following genes in LEGH: the GNAS, KRAS, and STK11 genes. Further, they observed that these mutations are mutually exclusive. These results suggest that LEGH is associated with the transformation and progression process to GMC. In line with these data, Shibata et al. (39) reported that hepatobiliary mucinous cystic neoplasms with abundant mucinous epithelium harbored KRAS mutations more frequently and show higher risk of malignant progression compared to those with poor intracytoplasmic mucin. Although identifying mutations in the LEGH tissue has been performed, genetic aberrations that initiate or accelerate progression from LEGH to $\mathrm{GMC}$ remain to be explored.

We also presented a scarce case of UEA with the MELF pattern of stromal invasion. The MELF pattern of invasion represents a very distinctive morphological pattern of myometrial invasion of endometrial endometrioid carcinomas (40). Even though a recent study has described MELF invasion pattern in endocervical adenocarcinoma (41), little information is available on the clinicopathological significance of the MELF invasion pattern in UEA. Since this pattern of invasion is not familiar to pathologists, for accurate diagnosis, they must know that the MELF invasion pattern can be observed in UEA. Many previous studies reported that the MELF invasion pattern is significantly related to more frequent lymphovascular invasion and isolated tumor lymph node metastasis, as well as poor prognosis of patients with endometrial carcinoma $(42,43)$. Espinosa et al. (44) observed that MELF-positive endometrioid carcinoma cases had more frequent lymph node metastases than those without MELF. Zinovkin et al. (45) reported that the survival rate of patients whose tumors show MELF invasion pattern was significantly lower when compared with MELF-negative patients. Sanci et al. (46) also documented that the presence of MELF invasion pattern was a substantial risk factor for detecting lymph node involvement in patients with grade 1-2 endometrial endometrioid carcinoma, and its effect on OS was significant. These data suggest that the MELF invasion pattern can be a potential risk factor for lymph node metastasis, and for appropriate management, pathologists need to not overlook the MELF invasion pattern when diagnosing UEA. However, prognostic implications of MELF invasion pattern have not been completely established. In studies involving low-grade endometrial endometrioid carcinoma (39), even though the MELF invasion pattern was significantly associated with larger tumor sizes, myometrial invasion greater than 50\%, advanced stages, lymphovascular invasion, and lymph node metastasis, patients who exhibited the MELF invasion pattern did not exhibit worse prognosis compared to those without the MELF invasion pattern. A recent study that analyzed the prognostic value of the MELF invasion pattern in a large cohort of patients with endocervical adenocarcinoma (41) demonstrated lower OS rates and shorter median survival in MELF-positive patients compared to MELF-negative patients. Regarding the insignificant adverse impact on patient outcomes, some previous studies on endometrial endometrioid carcinoma documented that the MELF component displayed very low proliferative activity or lack or Ki-67 labeling index and p21 expression $(40,47)$ compared to the areas of conventional invasion, indicating growth arrest or senescence of the MELF-type tumor element. The difference in the expression of proliferative and senescence markers may explain why the presence of MELF invasion pattern did not show any prognostic significance despite its association with frequent lymphovascular invasion and lymph node metastasis. Even though MELF was not independently associated with survival, previous data support the possibility that the pathological characteristics of MELF-positive tumors and MELF-negative tumors are different to each other. Therefore, although the prognostic value of the MELF invasion pattern in endocervical adenocarcinoma has not been fully established, pathologists should carefully determine the MELF invasion pattern in UEA (41). In addition, further studies are necessary to confirm the prognostic significance of this unusual pattern of invasion in UEA.

In conclusion, we demonstrated significant differences in clinicopathological and molecular characteristics between GMC and UEA. In comparison to UEA, GMC exhibited 
significantly deeper invasion depth, larger horizontal spread, and more advanced stage. In addition, the parametrial and vaginal extension with resection margin involvement and distant metastasis were more frequently observed in patients with GMC than in those with UEA. The disease-free survival time of GMC patients was significantly shorter than that of UEA patients. These findings confirm that GMC is a more aggressive subtype of endocervical adenocarcinoma than UEA, and that patient outcomes of GMC are worse than those of UEA. Mutually exclusive immunostaining patterns for p16 and p53 were found in UEA and GMC. All GMCs displayed the mutant p53 immunostaining pattern, whereas all UEAs exhibited wild-type p53 expression. In contrast, p16 expression was block-positive in all UEAs, but negative or patchy positive in GMCs, which suggests that the distinctive expression of p53 and p16 allows differentiating between UEA and GMC diagnoses. In addition, GMCs harbored pathogenic mutations in KRAS, TP53, NF1, STK11, $C D K N 2 A$, and ARID1A. Further, GMC also harbored MDM2 amplification. In contrast, UEAs exhibited different molecular profiles, in which mutations in HRAS, PIK3CA, and BRCA2 were harbored, which suggests that GMC and UEA exhibit genetic heterogeneity with potentially actionable molecular alterations.

\section{Conflicts of Interest}

The Authors have no conflicts of interest to declare regarding the study.

\section{Authors' Contributions}

All Authors made substantial contributions to the conception and design of the study; the acquisition, analysis, and interpretation of the data; drafting of the manuscript; critical revision of the manuscript for important intellectual content; and the final approval of the version to be published.

\section{Acknowledgements}

This research was supported by the Chungnam National University Hospital Research Fund (2019) and by the National Research Foundation of Korea (NRF) grant funded by the Korea government (MSIT) (2019R1G1A1100578).

\section{References}

1 Kurman RJ, Carcangiu ML, Herrington S and Young RH: WHO classification of tumours of female reproductive organs. IARC, 2014.

2 Bray F, Ferlay J, Soerjomataram I, Siegel RL, Torre LA and Jemal A: Global cancer statistics 2018: GLOBOCAN estimates of incidence and mortality worldwide for 36 cancers in 185 countries. CA Cancer J Clin 68: 394-424, 2018. PMID: 30207593. DOI: $10.3322 /$ caac. 21492
3 Mathew A and George PS: Trends in incidence and mortality rates of squamous cell carcinoma and adenocarcinoma of cervix - worldwide. Asian Pac J Cancer Prev 10: 645-650, 2009. PMID: 19827887.

4 Smith HO, Tiffany MF, Qualls CR and Key CR: The rising incidence of adenocarcinoma relative to squamous cell carcinoma of the uterine cervix in the United States: a 24-year population-based study. Gynecol Oncol 78: 97-105, 2000. PMID: 10926787. DOI: 10.1006/gyno.2000.5826

5 Visioli CB, Zappa M, Ciatto S, Iossa A and Crocetti E: Increasing trends of cervical adenocarcinoma incidence in Central Italy despite Extensive Screening Programme, 19852000. Cancer Detect Prev 28: 461-464, 2004. DOI: 10.1016/j.cdp.2004.07.007

6 Liu S, Semenciw R and Mao Y: Cervical cancer: the increasing incidence of adenocarcinoma and adenosquamous carcinoma in younger women. Can Med Assoc J 164: 1151-1152, 2001. PMID: 11338801.

7 Stolnicu S, Barsan I, Hoang L, Patel P, Terinte C, Pesci A, AvielRonen S, Kiyokawa T, Alvarado-Cabrero I, Pike MC, Oliva E, Park KJ and Soslow RA: International Endocervical Adenocarcinoma Criteria and Classification (IECC): a new pathogenetic classification for invasive adenocarcinomas of the endocervix. Am J Surg Pathol 42: 214-226, 2018. PMID: 29135516. DOI: $10.1097 /$ pas.0000000000000986

8 Kusanagi Y, Kojima A, Mikami Y, Kiyokawa T, Sudo T, Yamaguchi $\mathrm{S}$ and Nishimura R: Absence of high-risk human papillomavirus (HPV) detection in endocervical adenocarcinoma with gastric morphology and phenotype. Am J Pathol 177: 21692175, 2010. PMID: 20829441. DOI: 10.2353/ajpath.2010.100323

9 Garg S, Nagaria TS, Clarke B, Freedman O, Khan Z, Schwock J, Bernardini MQ, Oza AM, Han K, Smith AC, Stockley TL and Rouzbahman M: Molecular characterization of gastric-type endocervical adenocarcinoma using next-generation sequencing. Mod Pathol 32: 1823-1833, 2019. PMID: 31308508. DOI: 10.1038/s41379-019-0305-x

10 Bae GE, Do SI, Kim K, Park JH, Cho S and Kim HS: Increased sphingosine kinase 1 expression predicts distant metastasis and poor outcome in patients with colorectal cancer. Anticancer Res 39: 663670, 2019. PMID: 30711943. DOI: 10.21873/anticanres.13161

11 Jung YY, Woo HY and Kim HS: Targeted genomic sequencing reveals novel TP53 in-frame deletion mutations leading to p53 overexpression in high-grade serous tubo-ovarian carcinoma. Anticancer Res 39: 2883-2889, 2019. PMID: 31177126. DOI: 10.21873/anticanres.13417

12 Kim HS, Kim JY, Lee YJ, Kim SH, Lee JY, Nam EJ, Kim S, Kim SW and Kim YT: Expression of programmed cell death ligand 1 and immune checkpoint markers in residual tumors after neoadjuvant chemotherapy for advanced high-grade serous ovarian cancer. Gynecol Oncol 151: 414-421, 2018. PMID: 30314669. DOI: 10.1016/j.ygyno.2018.08.023

$13 \mathrm{Na} \mathrm{K}$ and Kim HS: Clinicopathological characteristics of fallopian tube metastases from primary endometrial, cervical, and nongynecological malignancies: a single institutional experience. Virchows Arch 471: 363-373, 2017. PMID: 28702779. DOI: $10.1007 / \mathrm{s} 00428-017-2186-\mathrm{z}$

$14 \mathrm{Na} \mathrm{K}$ and Kim HS: Clinicopathologic and molecular characteristics of mesonephric adenocarcinoma arising from the uterine body. Am J Surg Pathol 43: 12-25, 2019. PMID: 29189288. DOI: 10.1097/PAS.0000000000000991 
15 Na K, Sung JY and Kim HS: Stromal p16 Overexpression in adult granulosa cell tumors of the ovary. Anticancer Res 37: 2437-2444, 2017. PMID: 28476811. DOI: 10.21873/anticanres.11583

16 Na K, Sung JY and Kim HS: TP53 mutation status of tuboovarian and peritoneal high-grade serous carcinoma with a wildtype p53 immunostaining pattern. Anticancer Res 37: 66976703, 2017. PMID: 29187446. DOI: 10.21873/anticanres.12128

17 Sung JY, Jung YY and Kim HS: Clinicopathological characteristics and KRAS mutation status of endometrial mucinous metaplasia and carcinoma. Anticancer Res 38: 27792786, 2018. PMID: 29715099. DOI: 10.21873/anticanres.12521

18 Sung JY, Na K and Kim HS: Down-regulation of inositol polyphosphate 4-phosphatase type II expression in colorectal carcinoma. Anticancer Res 37: 5525-5531, 2017. PMID: 28982866. DOI: 10.21873/anticanres.11984

19 Na K, Lee JY, Sung JY, Kim GM, Koo JS and Kim HS: Comparative clinicopathological and cytomorphological analyses of peritoneal carcinomatosis associated with metastatic breast carcinoma and primary peritoneal/ovarian carcinoma in patients with a history of breast carcinoma. Virchows Arch 473: 165-175, 2018. PMID: 29926183. DOI: 10.1007/s00428-018-2390-5

20 Jung YY, Nahm JH and Kim HS: Cytomorphological characteristics of glassy cell carcinoma of the uterine cervix: histopathological correlation and human papillomavirus genotyping. Oncotarget 7: 74152-74161, 2016. PMID: 27708230. DOI: 10.18632/oncotarget.12361

21 Bae GE, Yoon G, Song YJ and Kim HS: High-grade squamous intraepithelial lesion arising adjacent to vulvar lymphangioma circumscriptum: a tertiary institutional experience. Oncotarget 7: 48120-48129, 2016. PMID: 27329721. DOI: 10.18632/oncotarget. 10158

$22 \mathrm{Na} \mathrm{K}$, Sung JY and Kim HS: clinicopathological characteristics of high-grade squamous intraepithelial lesions involving condyloma acuminatum. Anticancer Res 38: 1767-1774, 2018. PMID: 29491115. DOI: 10.21873/anticanres.12414

$23 \mathrm{Na} \mathrm{K}$, Kim HS, Shim HS, Chang JH, Kang SG and Kim SH: Targeted next-generation sequencing panel (TruSight Tumor 170) in diffuse glioma: a single institutional experience of 135 cases. J Neurooncol 142: 445-454, 2019. PMID: 30710203. DOI: $10.1007 / \mathrm{s} 11060-019-03114-1$

24 Karamurzin YS, Kiyokawa T, Parkash V, Jotwani AR, Patel P, Pike MC, Soslow RA and Park KJ: Gastric-type endocervical adenocarcinoma: an aggressive tumor with unusual metastatic patterns and poor prognosis. Am J Surg Pathol 39: 1449-1457, 2015. PMID: 26457350. DOI: 10.1097/PAS.0000000000000532

25 Nishio S, Mikami Y, Tokunaga H, Yaegashi N, Satoh T, Saito M, Okamoto A, Kasamatsu T, Miyamoto T, Shiozawa T, Yoshioka Y, Mandai M, Kojima A, Takehara K, Kaneki E, Kobayashi H, Kaku $\mathrm{T}$, Ushijima $\mathrm{K}$ and Kamura T: Analysis of gastric-type mucinous carcinoma of the uterine cervix - An aggressive tumor with a poor prognosis: a multi-institutional study. Gynecol Oncol 153: 13-19, 2019. PMID: 30709650. DOI: 10.1016/j.ygyno.2019.01.022

26 Kim YJ, Lee KJ, Park KR, Kim J, Jung W, Lee R, Kim SC, Moon HS, Ju W, Kim YH and Lee J: Prognostic analysis of uterine cervical cancer treated with postoperative radiotherapy: importance of positive or close parametrial resection margin. Radiat Oncol J 33: 109-116, 2015. PMID: 26157680. DOI: 10.3857/roj.2015.33.2.109

27 McCann GA, Taege SK, Boutsicaris CE, Phillips GS, Eisenhauer EL, Fowler JM, O'Malley DM, Copeland LJ, Cohn DE and Salani
R: The impact of close surgical margins after radical hysterectomy for early-stage cervical cancer. Gynecol Oncol 128: 44-48, 2013. PMID: 23138134. DOI: 10.1016/j.ygyno.2012.10.028

28 Kojima A, Mikami Y, Sudo T, Yamaguchi S, Kusanagi Y, Ito M and Nishimura R: Gastric morphology and immunophenotype predict poor outcome in mucinous adenocarcinoma of the uterine cervix. Am J Surg Pathol 31: 664-672, 2007. PMID: 17460448. DOI: 10.1097/01.pas.0000213434.91868.b0

29 Park KJ, Kim MH, Kim JK and Cho KS: Gastric-type adenocarcinoma of the uterine cervix: magnetic resonance imaging features, clinical outcomes, and prognostic factors. Int J Gynecol Cancer 28: 1203-1210, 2018. PMID: 29683881. DOI: 10.1097/IGC.0000000000001275

30 Carleton C, Hoang L, Sah S, Kiyokawa T, Karamurzin YS, Talia KL, Park KJ and McCluggage WG: A detailed immunohistochemical analysis of a large series of cervical and vaginal gastrictype adenocarcinomas. Am J Surg Pathol 40: 636-644, 2016. PMID: 26685087. DOI: 10.1097/PAS.0000000000000578

31 Talia KL and McCluggage WG: The developing spectrum of gastric-type cervical glandular lesions. Pathology 50: 122-133, 2018. PMID: 29233547. DOI: 10.1016/j.pathol.2017.09.009

32 Wada T, Ohishi Y, Kaku T, Aman M, Imamura H, Yasutake N, Sonoda $\mathrm{K}$, Kato $\mathrm{K}$ and Oda $\mathrm{Y}$ : endocervical adenocarcinoma with morphologic features of both usual and gastric types: clinicopathologic and immunohistochemical analyses and high-risk HPV detection by in situ hybridization. Am J Surg Pathol 41: 696-705, 2017. PMID: 28296678. DOI: 10.1097/PAS.0000000000000833

33 Garg S, Nagaria TS, Clarke B, Freedman O, Khan Z, Schwock J, Bernardini MQ, Oza AM, Han K, Smith AC, Stockley TL and Rouzbahman M: Molecular characterization of gastric-type endocervical adenocarcinoma using next-generation sequencing. Mod Pathol 32: 1823-1833, 2019. DOI: 10.1038/s41379-0190305-x

34 Feng C, Xian Q and Liu S: Micro RNA-518 inhibits gastric cancer cell growth by inducing apoptosis via targeting MDM2. Biomed Pharmacother 97: 1595-1602, 2018. PMID: 29793321. DOI: 10.1016/j.biopha.2017.11.091

35 Drakos E, Singh RR, Rassidakis GZ, Schlette E, Li J, Claret FX, Ford RJ, Jr., Vega F and Medeiros LJ: Activation of the p53 pathway by the MDM2 inhibitor nutlin-3a overcomes BCL2 overexpression in a preclinical model of diffuse large B-cell lymphoma associated with $\mathrm{t}(14 ; 18)(\mathrm{q} 32 ; \mathrm{q} 21)$. Leukemia 25 : 856-867, 2011. PMID: 21394100. DOI: 10.1038/leu.2011.28

36 Ruijs MW, Verhoef S, Rookus MA, Pruntel R, van der Hout AH, Hogervorst FB, Kluijt I, Sijmons RH, Aalfs CM, Wagner A, Ausems MG, Hoogerbrugge N, van Asperen CJ, Gomez Garcia EB, Meijers-Heijboer H, Ten Kate LP, Menko FH and van 't Veer LJ: TP53 germline mutation testing in 180 families suspected of LiFraumeni syndrome: mutation detection rate and relative frequency of cancers in different familial phenotypes. J Med Genet 47: 421428, 2010. PMID: 20522432. DOI: 10.1136/jmg.2009.073429

37 Garg K, Karnezis AN and Rabban JT: Uncommon hereditary gynaecological tumour syndromes: pathological features in tumours that may predict risk for a germline mutation. Pathology 50: 238256, 2018. PMID: 29373116. DOI: 10.1016/j.pathol.2017.10.009

38 Matsubara A, Sekine S, Ogawa R, Yoshida M, Kasamatsu T, Tsuda $\mathrm{H}$ and Kanai Y: Lobular endocervical glandular hyperplasia is a neoplastic entity with frequent activating GNAS mutations. Am J Surg Pathol 38: 370-376, 2014. PMID: 24145653. DOI: 10.1097/PAS.0000000000000093 
39 Shibata H, Ohike N, Norose T, Isobe T, Suzuki R, Imai H, Shiokawa A, Aoki T, Murakami M, Mizukami H, Tanaka JI and Takimoto M: Mucinous cystic neoplasms lined by abundant mucinous epithelium frequently involve KRAS mutations and malignant progression. Anticancer Res 37: 7063-7068, 2017. PMID: 29187496. DOI: 10.21873/anticanres.12178

40 Kihara A, Yoshida H, Watanabe R, Takahashi K, Kato T, Ino Y, Kitagawa $\mathrm{M}$ and Hiraoka N: Clinicopathologic association and prognostic value of microcystic, elongated, and fragmented (MELF) pattern in endometrial endometrioid carcinoma. Am J Surg Pathol 41: 896-905, 2017. PMID: 28418994. DOI: 10.1097/PAS.0000000000000856

41 Segura SE, Hoang L, Boros M, Terinte C, Pesci A, Aviel-Ronen S, Kiyokawa T, Alvarado-Cabrero I, Oliva E, Park KJ, Soslow RA and Stolnicu S: Clinicopathologic association and prognostic value of melf pattern in invasive endocervical adenocarcinoma (ECA) as classified by IECC. Int J Gynecol Pathol, 2019. PMID: 31517653. DOI: 10.1097/PGP.0000000000000633

42 Park JY, Hong D and Park JY: Association between morphological patterns of myometrial invasion and cancer stem cell markers in endometrial endometrioid carcinoma. Pathol Oncol Res 25: 123-130, 2019. PMID: 28990139. DOI: $10.1007 / \mathrm{s} 12253-017-0320-5$

43 Ruz-Caracuel I, Ramon-Patino JL, Lopez-Janeiro A, Yebenes L, Berjon A, Hernandez A, Gallego A, Heredia-Soto V, Mendiola M, Redondo A, Pelaez-Garcia A and Hardisson D: Myoinvasive pattern as a prognostic marker in low-grade, early-stage endometrioid endometrial carcinoma. Cancers (Basel) 11, 2019. PMID: 31766622. DOI: 10.3390/cancers11121845
44 Espinosa I, Serrat N, Zannoni GF, Rovira R, D’Angelo E and Prat J: Endometrioid endometrial carcinomas with microcystic, elongated, and fragmented (MELF) type of myoinvasion: role of immunohistochemistry in the detection of occult lymph node metastases and their clinical significance. Hum Pathol 70: 6-13, 2017. PMID: 28601659. DOI: 10.1016/j.humpath.2017.05.026

45 Zinovkin DA, Pranjol MZI, Petrenyov DR, Nadyrov EA and Savchenko OG: The potential roles of MELF-pattern, microvessel density, and VEGF expression in survival of patients with endometrioid endometrial carcinoma: a morphometrical and immunohistochemical analysis of 100 cases. J Pathol Transl Med 51: 456-462, 2017. PMID: 28934825. DOI: 10.4132/jptm.2017. 07.19

46 Sanci M, Gungorduk K, Gulseren V, Karadeniz T, Kocaer M, Gungorduk $\mathrm{O}$ and Ozdemir IA: MELF pattern for predicting lymph node involvement and survival in grade I-II endometrioid-type endometrial cancer. Int J Gynecol Pathol 37: 17-21, 2018. PMID: 28319574. DOI: 10.1097/PGP.0000000 000000370

47 Stewart CJ, Crook ML and Doherty DA: Micro-anatomical variation in cellular proliferation in endometrial adenocarcinoma, and inverse correlation between Ki67 and cytokeratin 7 expression. Histopathology 57: 46-54, 2010. PMID: 20557372. DOI: $10.1111 / \mathrm{j} .1365-2559.2010 .03588 . \mathrm{x}$

Received May 5, 2020

Revised May 23, 2020

Accepted June 22, 2020 